\title{
Crescimento e produtividade do crambe em resposta à calagem e ao espaçamento entre filleiras ${ }^{1}$
}

\author{
Rodrigo Gomes Branquinho², José Milton Alves ${ }^{3}$
}

Resumo: O crambe é uma espécie vegetal oleaginosa potencial fornecedora de matéria prima para biodiesel. No Brasil, seu cultivo é incipiente, sendo necessárias informações relativas ao manejo da cultura. Assim, o objetivo com este trabalho foi avaliar o efeito de quatro espaçamentos entre linhas $(0,15 ; 0,30 ; 0,45$; e $0,60 \mathrm{~m})$ e três níveis de calagem $\left(0 ; 1.500\right.$; e $3.000 \mathrm{~kg} \mathrm{ha}^{-1}$ de calcário) sobre o crescimento e produtividade da cultura do crambe. $\mathrm{O}$ experimento foi desenvolvido em condições de campo na área experimental de agroenergia do IF Goiano, campus Rio Verde, GO. Foram realizadas coletas e pesagens da parte aérea e das raízes 60 dias após a emergência, com a finalidade de acompanhar o crescimento das plantas. Por ocasião do final do ciclo da cultura, 77 dias após a emergência, as plantas da área útil, compreendidas em $1 \mathrm{~m}^{2}$, foram coletadas para a obtenção da produtividade de grãos, peso de 100 sementes e massa seca da parte aérea produzida por área. Dessas plantas, dez foram tomadas ao acaso para contagem do número de galhos. Os resultados indicaram que plantas com fileiras espaçadas em $0,60 \mathrm{~m}$ apresentaram maior crescimento individual que aquelas com fileiras espaçadas em $0,15 \mathrm{~m}$; porém, o espaçamento de $0,15 \mathrm{~m}$ propicia maior produção de parte aérea por área comparado ao espaçamento de $0,60 \mathrm{~m}$. A aplicação de 1500 $\mathrm{kg} \mathrm{ha}^{-1}$ de calcário propicia maior crescimento individual das plantas aos 60 dias após a emergência, comparado a aplicação de $3000 \mathrm{~kg} \mathrm{ha}^{-1}$ de calcário. Maior produtividade de grãos é conseguida com a aplicação de $3000 \mathrm{~kg} \mathrm{ha}^{-1}$ de calcário e espaçamento entre fileiras de $0,30 \mathrm{~m}$. As quantidades de calcário avaliadas, no entanto, não influenciaram na massa da parte aérea produzida por área.

Palavras chave: Crambe abyssinica Hochst; Fertilidade; Solo; Calcário.

\section{Growth and productivity of crambe in response to liming and row spacing}

\begin{abstract}
Crambe is a oilseed vegetable species that has the potential to provides raw material for biodiesel. In Brazil, its cultivation is incipient, requiring information related to the management of the culture. Thus, the objective of this work was to evaluate the effect of four spacing between lines $(0.15 \mathrm{~m} ; 0.30 \mathrm{~m} ; 0.45 \mathrm{~m}$; and $0.60 \mathrm{~m})$ and three liming levels (0 kg ha-1; 1,500 kg ha-1; and 3,000 kg ha-1 of limestone), on the growth and productivity of the crambe culture. The experiment was carried out under field conditions in the IF Goiano experimental agroenergy area, Rio Verde campus. Collections and weighing of aerial parts and roots were carried out 60 days after emergence, in order to monitor plant growth. At the end of the crop cycle, 77 days after emergence, plants from the useful area, comprised of $1 \mathrm{~m} 2$, were collected to obtain grain yield, weight of 100 seeds and dry matter of the aerial part produced by area. Of these plants, ten were taken at random to count the number of branches. The results indicated that plants with rows spaced at $0.60 \mathrm{~m}$ showed greater individual growth than those with rows spaced at $0.15 \mathrm{~m}$; however, the $0.15 \mathrm{~m}$ spacing provides greater aerial part production per area compared to the $0.60 \mathrm{~m}$ spacing. The application of $1500 \mathrm{~kg}$ ha-1 of limestone provides greater individual growth of plants at 60 days after emergence, compared to the application of $3000 \mathrm{~kg}$ ha-1 of limestone. Higher grain productivity is achieved with the application of $3000 \mathrm{~kg} \mathrm{ha}^{-1}$ of limestone and row spacing of $0.30 \mathrm{~m}$. The quantities of limestone evaluated, however, did not influence the mass of the aerial part produced by area.
\end{abstract}

Keywords: Crambe abyssinica Hochst; Fertility; Soil; Limestone.

${ }^{1}$ Submetido em 06/09/2020 e aprovado em 06/12/2020;

${ }^{2}$ Faculdade Quirinópolis, Quirinópolis, Goiás, Brasil; E-mail: rodrigogobr@bol.com.br (Autor correspondente) - ORCID: https://orcid.org/00000001-9369-8935;

${ }^{3}$ Instituto Federal Goiano, Rio Verde, Goiás, Brasil; E-mail: jmiltonalves@gmail.com - ORCID: https://orcid.org/0000-0002-2477-206X

Agropecuária Técnica, Areia-PB, v. 41, n. 3-4, p. 65-70, 2020

https://doi.org/10.25066/agrotec.v41i3-4.55010 


\section{Introdução}

A crescente preocupação com as mudanças climáticas, particularmente o aquecimento global, e a expectativa de uma possível escassez de petróleo no futuro, tem impulsionado a demanda por energia renovável a partir de matérias primas agropecuárias. É nesse contexto que o biodiesel se encontra inserido, e, com o atual incentivo dado a sua produção, a pesquisa tem se dedicado a encontrar e desenvolver novas culturas oleaginosas. Por suas características agronômicas, pelo alto teor de óleo nas sementes, por seu potencial econômico e industrial, e por ser cultivado num período do ano destinado a "safrinha", o crambe (Crambe abyssinica Hoechst) adquire elevado interesse como matéria prima para produção de biodiesel.

O crambe desenvolve-se melhor em solos com textura média a moderadamente fina, bem drenados, férteis, profundos e com boa capacidade de exploração de umidade (Oplinger et al., 1991). Ainda não existem padrões específicos de adubação, porém alguns estudos já foram conduzidos de forma a orientar aspectos relativos à adubação nitrogenada (Vasconcelos et al., 2019), bem como potássica e fosfatada (Alves et al., 2016; Chaves e Souza, 2016; Alves et al., 2019). A faixa de $\mathrm{pH}$ ideal está em torno de 5,8 (Pitol et al., 2010), mas não há informações sobre resposta da cultura à calagem no Brasil. O espaçamento entre linhas reduzido promove menor ramificação e maior uniformidade na maturação, e rendimentos satisfatórios foram observados com 0,15 e $0,90 \mathrm{~m}$ entre fileiras (Oplinger et al., 1991). No entanto, larguras de entre linhas com $0,20 \mathrm{~m}$ propiciam rendimentos mais elevados (Zoz et al., 2018), sendo recomendado estande final com 70 a 120 plantas $\mathrm{m}^{-2}$ (Pitol, 2007).

No Brasil, a cultura tem apresentado rendimento de grãos entre 1500 e $1900 \mathrm{~kg} \mathrm{ha}^{-1}$, os quais contém, aproximadamente, $38 \%$ de óleo, que proporciona um biodiesel de boa qualidade em razão de possuir elevado teor de ácidos graxos com alto peso molecular, podendo ainda ser usado na produção de plastificantes, náilon, lubrificantes (Martins et al., 2012).

A cultura do crambe vem adquirindo elevado interesse para a produção de biodiesel, e por ser a pesquisa, com essa oleaginosa, ainda incipiente, a cultura carece de informações que viabilizem o seu cultivo. Nesta pesquisa o objetivo foi avaliar o efeito de espaçamentos e calagem sobre 0 crescimento e produtividade do crambe.

\section{Material e Métodos}

$\mathrm{O}$ experimento foi conduzido em condições de campo na área experimental de agroenergia do Instituto Federal Goiano, campus Rio Verde $\left(17^{\circ}\right.$ $47^{\prime}$ 53" de Latitude Sul; $51^{\circ} 55^{\prime}, 53^{\prime \prime}$ de Longitude Oeste; e $730 \mathrm{~m}$ de altitude). Foi avaliado o efeito de quatro espaçamentos entre linhas $(0,15,0,30,0,45$ e $0,60 \mathrm{~m})$ e três níveis de calagem $\left(0,1.500\right.$ e $\left.3.000 \mathrm{~kg} \mathrm{ha}^{-1}\right)$, constando de um fatorial 4 x 3, com três repetições em delineamento de blocos ao acaso. As parcelas constaram de $4 \mathrm{~m}^{2}(2 \times 2 \mathrm{~m})$.

Antes da instalação do experimento, procedeuse a amostragem e análise do solo. As amostras foram encaminhadas para análise química (Tabela 1) e textural no laboratório de solos do IF Goiano. A análise física do solo, na camada de $0-0,20 \mathrm{~m}$ de profundidade, indicou textura argilosa $(51,88 \%$ de argila; $11,64 \%$ de silte; e $36,48 \%$ de areia).

Tabela 1 Resultado da análise química (fertilidade) de amostra do solo coletada na camada de $0-0,20 \mathrm{~m}$ retirada na área experimental

\begin{tabular}{|c|c|c|c|c|c|c|c|c|c|c|}
\hline $\begin{array}{c}\mathrm{P} \\
\mathrm{mg} \mathrm{dm}-3\end{array}$ & $\begin{array}{l}\text { M.O } \\
\mathrm{g} \mathrm{dm}^{-3}\end{array}$ & $\begin{array}{c}\mathrm{pH} \\
\text { em água }\end{array}$ & $\mathrm{K}^{+}$ & $\mathrm{Ca}^{++}$ & $\mathrm{Mg}^{++}$ & $\begin{array}{l}\mathrm{H}^{+}+\mathrm{Al}^{+++} \\
--\mathrm{mmol}_{\mathrm{c}} \mathrm{dm}\end{array}$ & $\begin{array}{c}S \\
-3\end{array}$ & $\mathrm{Al}^{+++}$ & $\mathrm{T}$ & V\% \\
\hline 1,85 & 44,32 & 6,80 & 2,81 & 40,40 & 11,60 & 56,10 & 54,81 & 0,00 & 110,91 & 49,42 \\
\hline
\end{tabular}

As quantidades de calcário utilizadas foram calculadas para os níveis de saturação por bases de $50 \%, 60 \%$ e $70 \%$, o qual foi incorporado à profundidade de $0,20 \mathrm{~m}$. Foi realizada uma adubação com superfosfato simples no sulco de semeadura, na dose de $80 \mathrm{~kg} \mathrm{ha}^{-1}$ de $\mathrm{P}_{2} \mathrm{O}_{5}$. As

Agropecuária Técnica, Areia-PB, v. 41, n. 3-4, p. 65-70, 2020

https://doi.org/10.25066/agrotec.v41i3-4.55010 
sementes utilizadas foram da variedade FMS Brilhante. A semeadura e a adubação foram feitas em sulco, com a colocação do adubo no fundo deste, incorporando-o e, em seguida, distribuindo 40 sementes por metro de sulco à profundidade de $2 \mathrm{~cm}$. A calagem, adubação e semeadura foram realizadas nos dias 15 e 16 de abril de 2008 . Quando as plantas completaram 10 dias de emergência, procedeu-se o raleio a fim que fosse mantida a quantidade de 25 plantas por metro linear em todas as parcelas, proporcionando o equivalente a 1.666.666, 833.333, 555.555 e 416.666 plantas por hectare sob os respectivos espaçamentos de $0,15,0,30,0,45$ e 0,60 .

O crescimento da cultura foi acompanhado através de amostragens das plantas com a obtenção da massa seca da parte aérea e das raízes aos 60 DAE. Foram coletadas aleatoriamente quatro plantas, utilizando uma pá como ferramenta, a fim de conservar íntegras as raízes. As plantas foram lavadas em água corrente, separadas parte aérea de raízes, e levadas para a estufa de secagem com circulação forçada, onde permaneceram até o peso constante.

Por ocasião do final do ciclo da cultura, 77 DAE, as plantas da área útil, compreendidas em 1 $\mathrm{m}^{2}$, foram coletadas para a obtenção da produtividade, do peso de 100 grãos e da massa seca da parte aérea produzida por área. Em cada unidade experimental, dez das plantas foram tomadas ao acaso para contagem dos galhos principais, metodologia também utilizada por Laghetti et al (1995) em trabalhos com a cultura do crambe. Os grãos foram manualmente separados da parte aérea, e a parte aérea das raízes, e, em seguida, todo material seguiu para estufa, onde permaneceu até o peso constante. Foi feita análise de variância e as médias foram comparadas pelo teste de Tukey ao nível de $5 \%$ de probabilidade de erro, utilizando o software SAEG 5.0 (Funarbe, 1993).

\section{Resultados e Discussão}

As observações de campo indicaram que em Rio Verde - GO, nas circunstâncias em que foi cultivado, o crambe leva cerca de 30 dias para florescer, contados a partir da emergência (Dias Após a Emergência - DAE), e 77 DAE para completar o seu ciclo. Portanto, a cultura antecipou o início do florescimento, estimado para 35 DAE (Pitol, 2008), e a maturação, estimada entre 90 a 110 DAE (Oplinger et. al., 1991). Isso pode ter acontecido como resultado da resposta adaptativa da cultivar FMS Brilhante à região em que foi cultivada, no município de Rio Verde $\mathrm{GO}$, bem como ao período do ano.

Aos 60 DAE, os maiores espaçamentos $(0,60 \mathrm{e}$ $0,45 \mathrm{~m}$ ) propiciaram produção de raízes e de parte aérea significativamente superior ao espaçamento de $0,15 \mathrm{~m}$, mas sem diferir de entre linhas espaçadas em 0,30 m (Tabela 2). Os níveis de calagem avaliados também influenciaram as variáveis massa seca da parte aérea e massa seca das raízes aos 60 DAE. A aplicação de 1500 $\mathrm{kg} \mathrm{ha}^{-1}$ de calcário propiciou crescimento da parte aérea e das raízes superior à testemunha sem calagem, mas sem diferir da aplicação de 3000 $\mathrm{kg} \mathrm{ha}^{-1}$ (Tabela 3).

Tabela 2 Efeito de espaçamento sobre a massa seca (g planta $^{-1}$ ) das raízes e da parte aérea da cultura do crambe aos 60 dias após a emergência

\begin{tabular}{ccc}
\hline Espaçamento $(\mathrm{m})$ & Raízes $(\mathrm{g})$ & Parte Aérea $(\mathrm{g})$ \\
\hline 0,15 & $0,250 \mathrm{~B}$ & $3,841 \mathrm{~B}$ \\
0,30 & $0,320 \mathrm{AB}$ & $4,853 \mathrm{AB}$ \\
0,45 & $0,384 \mathrm{~A}$ & $5,890 \mathrm{~A}$ \\
0,60 & $0,423 \mathrm{~A}$ & $6,640 \mathrm{~A}$ \\
\hline p-valor & 0,0007 & 0,0051 \\
\hline C.V. $(\%)$ & 23,05 & 29,12 \\
\hline
\end{tabular}

Médias seguidas das mesmas letras, nas colunas, não diferem estatisticamente entre si pelo teste de Tukey $(\mathrm{p} \leq 0,05)$.

Tabela 3 Efeito da calagem sobre a massa seca ( $\mathrm{g}$ planta $^{-1}$ ) das raízes e da parte aérea da cultura do crambe aos 60 dias após a emergência

\begin{tabular}{ccc}
\hline Calagem $\left(\mathrm{kg} \mathrm{ha}^{-1}\right)$ & Raízes & Parte Aérea \\
\hline 0 & $0,292 \mathrm{~B}$ & $4,430 \mathrm{~B}$ \\
1500 & $0,375 \mathrm{~A}$ & $6,139 \mathrm{~A}$ \\
3000 & $0,365 \mathrm{AB}$ & $5,350 \mathrm{AB}$ \\
\hline p-valor & 0,0357 & 0,0418 \\
\hline C.V. $(\%)$ & 23,05 & 29,12 \\
\hline
\end{tabular}

Médias seguidas das mesmas letras, nas colunas, não diferem estatisticamente entre si pelo teste de Tukey $(p \leq 0,05)$.

O número de galhos por planta, medida por ocasião do final do ciclo da cultura aos 77 DAE, foi influenciada pelos espaçamentos. Nesta 
avaliação, os espaçamentos de $0,60,0,45$ e $0,30 \mathrm{~m}$ propiciaram engalhamento de plantas superior ao menor espaçamento, $0,15 \mathrm{~m}$ (Tabela 4). Este resultado está de acordo com o sugerido por Oplinger et al. (1991) que aponta para menor ramificação em espaçamentos reduzidos. Os níveis de calagem avaliados não influenciaram significativamente a variável número de galhos.

Tabela 4 Massa seca da parte aérea $\left(\mathrm{kg} \mathrm{ha}^{-1}\right)$ e número de galhos por planta de crambe aos 77 dias após a emergência

\begin{tabular}{ccc}
\hline Espaçamento $(\mathrm{m})$ & Parte aérea & Número de galhos \\
\hline 0,15 & $1498 \mathrm{~A}$ & $6,844 \mathrm{~B}$ \\
0,30 & $1418 \mathrm{AB}$ & $9,067 \mathrm{~A}$ \\
0,45 & $1166 \mathrm{AB}$ & $9,156 \mathrm{~A}$ \\
0,60 & $1120 \mathrm{~B}$ & $10,800 \mathrm{~A}$ \\
\hline p-valor & 0,0187 & 0,0007 \\
\hline C.V. $(\%)$ & 21,14 & 18,74 \\
\hline
\end{tabular}

Médias seguidas das mesmas letras não diferenciam significativamente entre si pelo teste de Tukey $(\mathrm{p} \leq 0,05)$.

Com relação a variável massa seca da parte aérea, foi verificada diferença entre os espaçamentos, sendo que o menor espaçamento avaliado, $0,15 \mathrm{~m}$, foi superior ao maior espaçamento, 0,60 m (Tabela 4). Os níveis de calagem avaliados não influenciaram significativamente a massa seca da parte aérea produzida por área $(\mathrm{p}$-valor $=0,1207)$.

Para a produtividade dos grãos, na testemunha sem calagem, o espaçamento de $0,30 \mathrm{~m}$ foi superior ao menor espaçamento. No nível de calagem contendo $1500 \mathrm{~kg} \mathrm{ha}^{-1}$ de calcário, os espaçamentos de 0,15 e $0,60 \mathrm{~m}$ foram superiores ao espaçamento de $0,30 \mathrm{~m}$. Já no nível de calagem contendo $3000 \mathrm{~kg} \mathrm{ha}^{-1}$, não foi verificada diferença entre os espaçamentos sobre a produtividade dos grãos (Tabela 5).

Tabela 5 Produtividade e peso de 100 grãos de crambe

\begin{tabular}{|c|c|c|c|c|c|c|}
\hline \multirow{3}{*}{ Espaçamento (m) } & \multicolumn{3}{|c|}{ Produtividade $\left(\mathrm{kg} \mathrm{ha}^{-1}\right)$} & \multicolumn{3}{|c|}{ Peso de 100 grãos (g) } \\
\hline & \multicolumn{6}{|c|}{ Calagem $\left(\mathrm{kg} \mathrm{ha}^{-1}\right)$} \\
\hline & 0 & 1500 & 3000 & 0 & 1500 & 3000 \\
\hline 0,15 & $576 \mathrm{Bb}$ & $1078 \mathrm{Aa}$ & $693 \mathrm{Aab}$ & $0,761 \mathrm{Aa}$ & $0,729 \mathrm{Aa}$ & $0,662 \mathrm{Aa}$ \\
\hline 0,30 & $1088 \mathrm{~A} \mathrm{a}$ & $455 \mathrm{Bb}$ & $1130 \mathrm{Aa}$ & $0,748 \mathrm{Aa}$ & $0,713 \mathrm{Aa}$ & $0,744 \mathrm{Aa}$ \\
\hline 0,45 & $785 \mathrm{ABa}$ & $909 \mathrm{ABa}$ & $721 \mathrm{Aa}$ & $0,762 \mathrm{Aa}$ & $0,756 \mathrm{Aa}$ & $0,775 \mathrm{Aa}$ \\
\hline 0,60 & $685 \mathrm{ABa}$ & $1045 \mathrm{Aa}$ & $718 \mathrm{Aa}$ & $0,749 \mathrm{Aa}$ & $0,745 \mathrm{Aa}$ & $0,715 \mathrm{Aa}$ \\
\hline p-valor $(E \times C)$ & \multicolumn{3}{|c|}{0,0257} & \multicolumn{3}{|c|}{0,0383} \\
\hline C.V. $(\%)$ & \multicolumn{3}{|c|}{26,94} & \multicolumn{3}{|c|}{7,18} \\
\hline
\end{tabular}

Médias seguidas das mesmas letras, minúsculas nas linhas e maiúsculas nas colunas, não diferem entre si pelo teste de Tukey ( $\mathrm{p} \leq$ 0,05). E x C é a interação Espaçamento x Calcário.

Quanto à calagem, no espaçamento de $0,15 \mathrm{~m}$, o tratamento contendo $1500 \mathrm{~kg} \mathrm{ha}^{-1}$ de calcário foi significativamente superior à testemunha sem calagem (Tabela 5). Já no espaçamento de 0,30m, a testemunha sem calagem e o tratamento contendo $3000 \mathrm{~kg} \mathrm{ha}{ }^{-1}$ de calcário foram significativamente superiores ao tratamento contendo $1500 \mathrm{~kg} \mathrm{ha}^{-1}$ de calcário. Nos demais espaçamentos, 0,15 e $0,60 \mathrm{~m}$, não foi verificado efeito significativo dos níveis de calagem avaliados sobre a produtividade dos grãos. Quanto à variável peso de 100 grãos, não foi verificado efeito significativo dos espaçamentos e dos níveis de calagem avaliados.

No geral, os resultados indicaram que o maior espaçamento, $0,60 \mathrm{~m}$, propiciou maior desenvolvimento individual das plantas relativamente ao menor espaçamento, $0,15 \mathrm{~m}$. Porém, a massa da parte aérea produzida por área apresentou aumento significativo quando os espaçamentos entrelinhas foram reduzidos para $0,15 \mathrm{~m}$, indicando que a produtividade de matéria seca da parte aérea pode aumentar à medida que os ganhos pelo maior número de plantas na área superarem as perdas pelo menor desenvolvimento individual das plantas.

Com relação ao peso dos grãos, não foi verificado efeito significativo dos espaçamentos e dos níveis de calagem avaliados sobre essa característica. Quanto ao rendimento de grãos, são 
reportadas produtividades maiores em espaçamentos reduzidos. Zoz et al. (2018) constataram que, sob condições de boa disponibilidade de água, a cultura alcança produtividades maiores $\left(\sim 1760 \mathrm{~kg} \mathrm{ha}^{-1}\right) \quad \mathrm{com}$ espaçamento entre fileiras de $0,20 \mathrm{~m}$. Oliveira et al. (2020) também observaram que, com $0,20 \mathrm{~m}$ entre fileiras, o rendimento de grãos supera a proporcionado pelo maior espaçamento, de $0,40 \mathrm{~m}$, alcançando 2000 a $5500 \mathrm{~kg} \mathrm{ha}^{-1}$, a depender do fornecimento de adubo nitrogenado.

Alves et al. (2019) observaram que o crambe se desenvolve e alcança maiores produtividades de grãos quando a saturação por bases está em torno de $50 \%$. Entretanto, neste experimento, embora verificada diferença significativa entre os níveis de calagem avaliados, os resultados de produtividade não foram conclusivos. Cabe mencionar que no período de enchimento dos grãos nenhuma chuva foi verificada, podendo, isto, ter influenciado no resultado.

\section{Conclusões}

Plantas com fileiras espaçadas em $0,60 \mathrm{~m}$, apresentam maior crescimento individual que fileiras espaçadas em $0,15 \mathrm{~m}$, porém a massa da parte aérea produzida por área é maior no espaçamento de $0,15 \mathrm{~m}$;

A aplicação de $1500 \mathrm{~kg} \mathrm{ha}^{-1}$ de calcário propicia maior crescimento individual das plantas do que a aplicação de $3000 \mathrm{~kg} \mathrm{ha}^{-1}$ de calcário. No entanto, os níveis de calagem avaliados não influenciam na massa da parte aérea produzida por área;

Maior produtividade de grãos é conseguida com aplicação de $3000 \mathrm{~kg} \mathrm{ha}^{-1}$ de calcário e espaçamento entre fileiras de $0,20 \mathrm{~m}$.

\section{Referências}

Alves, J. M.; Leandro, W. M.; Alves, C. C. F.; Carlos, L.; Ribon, A. A.; Fernandes, K. L. Crambe dry matter and yield under doses of phosphorus and base saturation in the Cerrado of Goiás. Revista Brasileira de Engenharia Agrícola e Ambiental, v. 20, n. 5, p. 421-426, $2016 . \quad$ http://dx.doi.org/10.1590/18071929/agriambi.v20n5p421-426
Alves, J. M.; Leandro, W. M.; Costa, A. M.; Silva, P. O.; Alves, C. C. F. Calagem e adubação potássica no crescimento e produtividade de Crambe abyssinica Hortsh Ex. R. T. (Brassicaceae). Rio Verde-GO, Global Science and Technology, v. 12, n. 1, p. 30-42, 2019. https://rv.ifgoiano.edu.br/periodicos/index.php/gst/article/vi ew/1065/640

Chaves, L. H. G.; Souza, R. S. Doses de fósforo e potássio no desenvolvimento da cultura de Crambe Abyssinica. Revista Verde de Agroecologia e Desenvolvimento Sustentável, v. $11, \quad$ n. 2, p. 71-75, 2016. https://doi.org/10.18378/rvads.v11i2.4298

FUNARBE. SAEG - Sistema para análises estatísticas - versão 5.0. Viçosa: Fundação Arthur Bernardes. $1993 . \quad 80 \mathrm{p}$. http://arquivo.ufv.br/saeg/JoseIvo.htm

Laghetti, G.; Piergiovanni, A. R.; Perrino, P. 1995. Yield and oil quality in selected lines of Crambe abyssinica Hochst. Industrial Crops and Products. v. 4, n. 3, p. 203-212, 1995. https://doi.org/10.1016/0926-6690(95)00033-9

Martins, L. D.; Costa, F. P.; Lopes, J. C.; Rodrigues, W. N. Influence of pre-germination treatments and temperature on the germination of crambe seeds (Crambe abyssinica Hochst). Idesia (Arica), v. 30, n. 3, p. 23-28, 2012. http://dx.doi.org/10.4067/S0718-34292012000300003

Oliveira, L. D. de, Chaves, L. H. G., Cavalcante, A. R., de Souza, F. G., Vasconcelos, A. C. F. de, \& Araujo, J. S. Production components of crambe in paraiba semi-arid region with nitrogen fertilization and different spacings. Bioscience Journal, v. 36, n. 4, p. 1203-1210, 2020. https://doi.org/10.14393/BJ-v36n4a2020-48042

Oplinger, E.S.; Oelke, E.A.; Kaminski, A.R.; Putnam, D.H.; Teynor, T.M.; Doll, J.D.; Kelling, K.A.; Durgan, B.R.; e Noetzel, D.M. 1991. Alternative Field Crops Manual. http://www.hort.purdue.edu/newcrop/afcm/crambe.html. Acesso em 06 de setembro de 2020.

Pitol, C. 2007. Biodiesel: Culturas, Sistemas de Produção e Rotação de Culturas. http://www.fundacaoms.org.br Acesso em 22 de setembro de 2018. 
Pitol, C. 2008. Cultura do Crambe. Disponível em: http://www.fundacaoms.org.br Acesso em: 06 de setembro de 2020.

Pitol, C.; BROCH, D.L.; ROSCOE, R. Crambe: Tecnologia e Produção. Maracaju: Ed. Fundação MS, 2010, 60 p.

Vasconcelos, A.C.F.; Souza, L.H.G.CG.; Cavalcante, A.R.; Oliveira, L.D. Desenvolvimento de crambe sob estresse hídrico e doses de nitrogênio. IN.: Semana Oficial da
Engenharia e da Agronomia, 76, Palmas, Congresso Técnico Científico da Engenharia e da Agronomia, Palmas, 2019.

Zoz, T.; Steiner, F.; Zoz, A.; Castagnhara, D. D.; Witt, T. W.; Zanotto, M. D.; Auld, D. L. Effect of row spacing and plant density on grain yield and yield components of Crambe abyssinica Hochst. Londrina, Semina: Ciências Agrárias, v. $39, \quad$ n. $1, \quad$ p. 393-402, 2018. https://doi.org/10.5433/1679-0359.2018v39n1p393 